Abstract submitted to the $42^{\text {nd }}$ International Conference on Environmental Systems Hilton San Diego Resort and Spa; San Diego, California 15-19 July 2012

\title{
Numerical Study of Ammonia Leak and Dispersion in the International Space Station
}

\author{
Chang H. Son, The Boeing Company, Houston, TX, USA
}

\begin{abstract}
$\underline{\text { Abstract }}$
Management of off-nominal situations on-board the International Space Station (ISS) is important to its continuous operation. One situation of concern is an accidental release of a chemical into the ISS atmosphere. In particular, introduction of ammonia into the cabin atmosphere can occur via the interface heat exchangers (IFHX) between the external thermal control system containing ammonia and internal thermal control system that uses water as a coolant to remove heat from ISS subsystems. Breach of the water/ammonia barrier of the IFHX can lead to a catastrophic rupture. Once the liquid water/ammonia mixture exits the ITCS, it instantly vaporizes and mixes with the U.S. Laboratory cabin atmosphere that results in rapid contamination of the cabin. The goal of the study is to assess the amount of ammonia in the Russian Segment by the time the crew is able to isolate the U.S. Segment. A Computational Fluid Dynamics (CFD) model for an accurate prediction of airflow and ammonia transport in the frozen flow field within the assembly complete ISS cabin was developed. The localized effects of ammonia dispersion are examined and discussed.
\end{abstract}

